\title{
La formation aux ordres sociaux et à la délibération éthique : le praticien réflexif et la gestion de problèmes complexes...
}

\author{
Par Jeanne Simard et Marc-André Morency \\ Université du Québec à Chicoutimi
}

L'horizon complexe auquel font face les décideurs contemporains semble défier toute capacité de délibération sur le plan individuel ou sur le plan collectif. L'accumulation de problèmes et de nuages sombres de diverses natures fait même naître une souffrance diffuse mêlée d'appréhension, d'incompréhension, à la mesure même de la méconnaissance des sphères normatives impliquées. Le malaise s'accompagne à la limite d'un profond sentiment d'aliénation. Comment alors naviguer dans de telles eaux? Comment piloter nos navires, disent les cybernéticiens? Sera-t-il possible de sortir du paradigme inégalitaire et profondément aveugle que postule la loi du marché chère aux forces mercantiles? Jacques Attali estime pour sa part que la crise financière mondiale constitue déjà un tsunami dont on ne pourra s'extirper, vers 2010, qu'à la condition de mieux réguler la sphère financière, de faire cesser ce qu'il présente, avec de nombreux autres analystes, comme la capture des régulateurs actuels par certains acteurs de la financiarisation.

Ainsi, dans le monde actuel, les traumatismes subis par les collectivités, les régions, les pays, renvoient très souvent à un ensemble de rapports sociaux qui abrogent les souverainetés établies, le droit, et bousculent les autres sources normatives. Les problèmes, de ce fait, se trouvent gérés tant bien que mal et le plus souvent en mode correctif, a posteriori, par de multiples organisations multilatérales, voire par des forums plus ou moins formels (comme celui de Davos), et ce, dans le cadre d'un droit international encore très faiblement structuré. La règle de droit, l'État de droit, faisant souvent défaut, les problèmes locaux se trouvent gérés, comme l'a décrit l'économiste Hernando de Soto (2005), dans le contexte d'arrangements légaux, mais surtout extralégaux, coutumiers, particulièrement contraignants et ce, pour l'immense majorité des habitants de notre planète. Le statut encore incertain du droit de propriété n'est que l'un des problèmes soulignés dans la littérature traitant des obstacles au développement. De nombreux intervenants travaillent précisément à parer certains effets de cette fragilité des institutions ou encore à rectifier quelques lacunes observables. On obéit alors, sans le savoir, au précepte confucéen: "Gouverner, c'est rectifier. » Mais ce précepte ne nous paraît guère susceptible de conduire à un décodage en profondeur du mouvement des choses ni à s'y impliquer de façon substantielle. On fait alors le lit d'expédients destinés à la conservation des choses et des structures, plutôt que de construire une scène dynamique de gouvernance et de régulation.

Quelle voie suivre et créer à la fois, étant gestionnaire, pour maitriser la gestion de ces problèmes complexes ? Comment l'enseignement de la gestion peut-elle préparer les futurs gestionnaires à affronter l'incertain des circonstances et des partenaires, l'inconnu de nouvelles situations émergentes. Le gestionnaire public ou privé, s'il doit posséder des qualités sur le plan de l'analyse critique, de l'intervention dans des horizons forcément différents, comment doit-il être préparé ? 


\section{Le praticien réflexif et le cycle décisionnel}

Dans ce contexte très global où l'innovation devra être la règle, le futur praticien du monde des affaires doit apprendre beaucoup plus que des recettes utilitaristes, données telles quelles par les coutumes ou créées sans précaution par un engineering social restreint. On attend de lui beaucoup plus que la simple exécution, par exemple, des recettes d'un John Williamson (l'économiste à l'origine du Consensus de Washington). Il ne peut plus se contenter de demeurer dans une bulle étroite, animé par ses seules idées ou idoles favorites. Il doit discerner favorablement certaines valeurs traditionnelles garantes de la survie des sociétés et se faire le promoteur de nouvelles valeurs de développement répondant aux problèmes sans précédents qui les confrontent. Perrot, qui adhère à ces vues, souligne que :

La science de la gestion quitte de plus en plus les rives, sûres mais fausses, des modèles prévisionnels ou l'avenir est déjà inscrit en filigrane dans les relations actuelles clairement repérées pour aborder aux rivages incertains de l'herméneutique.

Par herméneutique, on doit comprendre la capacité d'interpréter, de discerner, de critiquer, propre à la prise de décision, à l'intervention innovante. Cette capacité s'inscrit de fait dans une séquence décisionnelle en forme de boucle récursive qui déborde la perception spontanée des situations, fait appel aux disciplines critiques pour échapper à la vision routinière et produire de nouveaux aperçus, ce qui nourrit ensuite une intervention susceptible de corriger certains éléments de la situation problématique. Cette présentation très simplifiée fait ressortir l'existence et l'interaction de deux champs d'action: le champ des pratiques et le champ de la réflexion théorique.

$\mathrm{Au}$ sein du champ des pratiques, l'acteur social se comporte le plus souvent en conformité aux usages courants dans son environnement. Cette vie courante constitue son immédiat. Il faut un effort persistant et guidé pour accéder à un regard empreint de distance critique par rapport au monde de l'immédiat. Les sciences ont dû batailler ferme pour concevoir, conserver et propager des épistémologies, des méthodes, des techniques de calcul pour renverser l'imaginaire commun. L'image de la «terre plate », du mouvement apparent du soleil et des planètes furent longues à céder la place aux lois de Kepler, à la vision actuelle d'une terre (Gaïa) polymorphe et matériellement bien structurée, au sein d'un Cosmos évolutif. Ce n'est que munie de tels nouveaux concepts (d'un immédiat expliqué) que l'intervention peut enfin devenir possible sur le monde habituel, dans un cadre citoyen, et non simplement comme objet de technicité particulière (génétique, économique, physique, etc.).

L'adepte de ce processus décisionnel créateur, un nouveau type de personnage d'influence, nous l'appelons, après Daniel Schön, le praticien réflexif. On peut assimiler les fonctions d'un tel leader, dont la figure plus générale est celle de l'intervenant, à celles que l'on retrouve dans divers domaines de l'action sociale. Ces pratiques, on les trouve modélisées, par exemple, dans la théorie de la recherche-action du psychosociologue Kurt Lewin, dans la praxéologie de Jean-Louis Le Moigne ou encore dans les études empiriques du psychologue social et philosophe Lawrence Kohlberg, qui a su décrire les trois et même quatre niveaux de conscience du praticien réflexif. 


\section{Quelques cas illustrant l'appel à un nouveau cadre décisionnel, à la pratique réflexive}

Cet appel au dépassement, à la pratique réflexive, à la délibération éthique des gestionnaires, surgit de partout. Les législateurs, les tribunaux, les ordres professionnels, les universités et même les institutions mondiales, appellent de plus en plus dépasser la logique de simple compliance aux normes ou au seul respect des pratiques traditionnelles ou convenues, soulignant le besoin de contributions éthiquement réfléchies de la part des diverses parties prenantes de la gouvernance. Voyons quelques exemples.

\section{Le gouvernement québécois, en avril} 2006, a affirmé clairement sa volonté de restructurer la gouvernance de l'administration publique

\section{Les législateurs canadien et québécois}

Le gouvernement québécois, en avril 2006, a affirmé clairement sa volonté de restructurer la gouvernance de l'administration publique, ce qui touche plus de 150 ministères, organismes et entreprises d'État. Dans l'esprit de la Déclaration de Rio sur l'environnement et le développement de 1992, le législateur québécois adoptait alors la Loi sur le développement durable. Cette loi affirme ou réaffirme certains principes directeurs tels l'équité intra et inter générationnelle, la conservation, la protection et le rétablissement de la santé des écosystèmes, la participation du public, la responsabilité environnementale, la prévention, la précaution, la responsabilité du pollueur/utilisateur-payeur, l'internalisation des coûts. Il introduisait aussi dans la Charte québécoise des droits et libertés de la personne, le droit de chacun «de vivre dans un environnement sain et respectueux de la biodiversité (art. 46.1) ${ }^{1}$.

Ce nouveau langage impose une nouvelle façon de comprendre les rapports qu'entretiennent les êtres humains entre eux et avec la nature. Les organisations publiques et les organisations privées qui voudront contracter avec l'administration québécoise ou recevoir de celleci des subventions, devront respecter ces principes qui appellent à une stratégie de gouvernance plus respectueuse de la qualité de l'environnement et de l'équité sociale. On devra intégrer, de manière opérationnelle, le développement durable dans la gouvernance des organisations. Cette technique d'intervention s'apparente au Compact des Nations Unies qui guide cette organisation dans ses rapports avec les entreprises désireuses de contracter avec elle.

$\underline{\text { Les tribunaux }}$

Les tribunaux canadiens ont rendu des jugements importants qui illustrent bien toute la complexité dans laquelle les gestionnaires doivent désormais opérer et décider.

\section{Prise en compte des parties prenantes}

En 2004, la Cour suprême du Canada a causé tout un émoi dans l'affaire People c. Wise en réaffirmant que selon la Loi canadienne sur les sociétés par actions (LCSA), la société par actions poursuit des objectifs qui lui sont propres et qui ne se limitent pas simplement à satisfaire les seuls intérêts des actionnaires (la doctrine Revlon... chère aux Américains). Ainsi, la Cour retient que les intérêts de la société par actions ne se confondent pas avec ceux des actionnaires, des créanciers ni avec ceux de quelque partie intéressée. En d'autres termes, les administrateurs d'une société par actions ne sont pas les fiduciaires d'intérêts particuliers, de créanciers ou d'autres parties prenantes de la société. Cette mise en garde fait ressortir que l'intérêt de la société par actions comme personne morale a été définie par la LCSA comme la valeur suprême encadrant ses rapports avec les autres entités qui composent son environnement. Son existence et son développement en tant que «meilleure entreprise » représentent le critère prééminent pour ses dirigeants. 
Par conséquent, on peut croire que cette décision de la Cour, et l'interprétation qu'elle fait de l'article 122 (1) (a) de la LCSA, sensibiliseront davantage les gestionnaires à la gouvernance, à la responsabilité sociale des entreprises, à l'éthique des affaires, au respect des déontologies professionnelles, au respect des lois en général, au Canada et à l'étranger. Cette interprétation plus précise de l'obligation fiduciaire aura certainement le mérite de faire disparaître l'épée de Damoclès qui semblait s'opposer à toute initiative de prise en compte des intérêts des autres parties prenantes de la société par actions, en atténuant la crainte de poursuites éventuelles. L'organisation peut, dans son intérêt même, prendre en compte une panoplie de préoccupations, réaliser son devenir souhaitable, sans que les administrateurs aient failli à leur devoir fiduciaire, bien au contraire. Un tel niveau de complexité rendra plus difficile le recrutement de bons administrateurs au sein des conseils d'administration; en particulier depuis l'adoption des lois du type SarbanesOxley, les entreprises américaines ont du mal à retenir ou à mobiliser des agents fiduciaires capables d'opérer à ce niveau de complexité, en échange des rémunérations courantes.

Prise en compte des droits ancestraux des autochtones

Pour définir la substance et la portée précise des droits ancestraux des autochtones, la Cour suprême, dans plusieurs de ses jugements, comprenant que ces questions ne peuvent trouver résolution seulement dans des arrêts de cour, des litiges, des confrontations, recommande fortement aux parties de s'engager dans une importante démarche de négociation et de faire appel à des notions comme la réflexion, la créativité, le respect mutuel, le partage des valeurs, le rapprochement culturel et la coexistence harmonieuse. La Cour suprême souhaite en quelque sorte que l'on entame à la grandeur du pays des processus collectifs de délibération éthique, des processus de création sous l'empire de contraintes historiques, religieuses, anthropologiques, sociologiques, juridiques, financières, etc. Ces processus exigent de connaittre les rapports dynamiques entre les divers ordres sociaux, supposent des changements dans les mentalités et les comportements.

On dénombre en fait actuellement quelque 600 revendications émanant des peuples autochtones au Canada. On peut supposer qu'autant de tables de négociation ont été mises en place au Québec et dans les autres parties du Canada afin d'en arriver à des ententes et à des traités pouvant opérationnaliser cette nouvelle approche de reconnaissance et de partage mutuels entre nos communautés. On sortirait enfin des approches conflictuelles, dominatrices, génocidaires, assimilatrices, des siècles passés, pour entrer dans un vivre-ensemble construit dans l'esprit de la négociation coopérative chère au théoricien de la négociation Gérard Nierenberg ou au politologue Robert Axelrod.

C'est ainsi qu'il faut comprendre la complexité de la gestion des projets qui se développent actuellement ou se développeront dans le Nord québécois. Pour les entreprises publiques, privées ou en partenariat (les PPP), le développement des activités minières, hydroélectriques, forestières, touristiques et celles de la construction, passera dorénavant par une association étroite et plus égalitaire avec les peuples Cri, Inuit et Innus.

\section{$\underline{\text { Prise en compte de la diversité culturelle }}$}

Dans le rapport de la commission BouchardTaylor sur les accommodements raisonnables, on recommande aux gestionnaires d'institutions publiques d'intensifier leurs efforts favorisant l'implantation de l'approche dite contextuelle, délibérative et réflexive. Peu de décisions sont susceptibles de requérir des accommodements raisonnables au sens de la loi. Par contre, les possibilités de mieux prendre en compte les différences culturelles et les valeurs consacrées par les chartes, seront innombrables et exigeront beaucoup de créativité. Ce genre de situation ne pourra aller qu'en augmentant avec l'avènement d'une société d'envergure planétaire. 


\section{$\underline{\text { Réflexions au sein des ordres professionnels }}$}

Les professionnels se voient généralement reconnaître, en vertu même de leur ordre social particulier, une assez grande autonomie. Par conséquent, ils se voient reconnaître un espace considérable de liberté d'expression et de délibération éthique. À titre d'exemple, l'article 5 du Code de déontologie des administrateurs agréés du Québec spécifie que :

Dans l'exercice de sa profession, l'administrateur agréé doit tenir compte de l'ensemble des conséquences prévisibles que peuvent avoir ses recherches, travaux ou interventions sur la société (notre souligné).

Cet article représente un engagement des professionnels de la gestion sur la base d'un principe ouvert de responsabilité et de loyauté à l'endroit des parties prenantes du corps social ambiant. Il s'agit là d'un fait accepté et ratifié par l'organe représentant le législateur, l'Office des professions. L'administrateur agréé doit en quelque sorte prévoir les impacts de sa pratique dans son milieu plus ou moins immédiat. Il ne lui suffit plus de s'interroger sur la conformité à une démarche préétablie, la simple conformité à une procédure ou à un ensemble déjà déterminé de règles. On a inscrit dans cet article 5 l'obligation, le devoir de faire l'éthique, de contribuer au progrès social. Il en va de l'honneur de la profession, de la protection du public. L'administrateur agréé, qu'il soit salarié ou consultant d'une organisation, peut alors pleinement participer à la définition du développement de l'organisation ou de l'entreprise et considérer la contribution (ou l'impact des décisions) de chacune des parties prenantes touchées par la décision. La déontologie propulse l'effort de délibération, et donc la réflexivité, jusqu'au palier de l'éthique appliquée, au sein d'une conjoncture particulière. Il est significatif de relever que les travailleurs sociaux, les agronomes, les urbanistes et les ingénieurs ont aussi incorporé à leur code de déontologie une mesure analogue d'ouverture à l'éthique, par des articles similaires à l'article 5 du Code des administrateurs agréés du Québec.

Réflexions au sein d'autres facultés ou départements

Les facultés de droit sont souvent présumées conservatrices. Mais est-ce bien le cas ? La plus importante au Canada, la Faculté de l'Université de Montréal, s'est résolument engagée dans une démarche interdisciplinaire. Le doyen Gilles Trudeau explique : «Nous excellons ici en droit positif, que nous analysons et critiquons, mais nous regardons aussi le phénomène juridique à travers le prisme des sciences sociales. L'interdisciplinarité est une tendance lourde, principalement en Amérique du Nord.» Quand on connaît l'influence du droit dans la gouverne des sociétés et des entreprises, il y a là une tendance exemplaire.

Un exemple d'intervention au niveau du système $\underline{\text { mondial }}$

La critique de Muhammad Yunus, prix Nobel de la paix de 2006, à l'endroit du système de crédit bancaire préside au développement d'un système mutualiste parallèle de financement dans diverses parties du monde, même aux ÉtatsUnis. Avec la crise du crédit sans précédent que ce pays traverse, on peut présumer que la méthode Yunnus du microcrédit fera figure de révolution.

\section{Nouveaux outils à employer dans la formation et dans la pratique}

Afin que les étudiants en gestion puissent mieux « résister aux sirènes de l'opinion commune », dépasser les représentations habituelles, voire la vision étriquée d'une simple éthique personnelle du gestionnaire, il est impératif de resituer le cadre corporatif dans l'univers institutionnel et dans son milieu social; l'étude de disciplines telles que le droit, 
la sociologie et l'éthique appliquée prend alors toute son importance dans la formation. La pratique réflexive requiert que le gestionnaire puisse reconnaître la nature très diversifiée des ordres sociaux (droit national et international, contrats, ordres religieux, déontologie, règlements des organisations, traditions, coutumes, règles de politesse, morale, etc.) pouvant concourir à donner un sens entier à son intervention. Le gestionnaire de haut niveau doit maîtriser les formes et le contenu du dialogue internormatif, lorsqu'il intervient auprès d'un client, d'un groupe, d'une organisation, bref quand il agit au sein d'un processus éthique de cocréation sujette à des contraintes.

C'est dans un tel objectif de formation qu'il nous est apparu qu'un tableau soigneusement structuré et documenté des sources normatives à l'œuvre serait un des moyens analytiques permettant de se représenter le champ des possibles et des contraintes. Nous achevons présentement un texte de synthèse sur les ordres sociaux, dans le but d'en faciliter l'enseignement. Nous en présentons ici le tableau schématique :

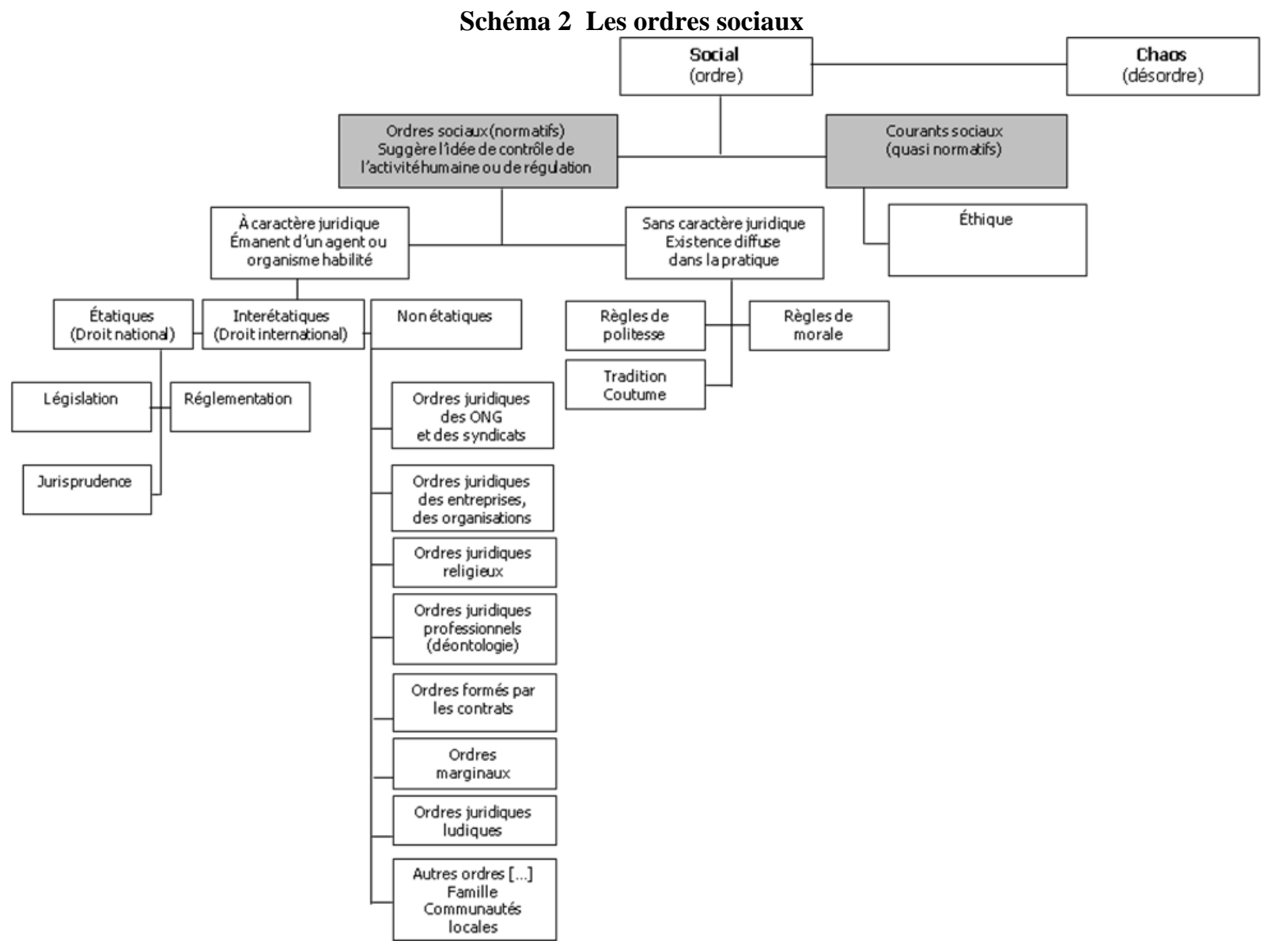

L'expérience nous a montré que l'enseignement de cette complexité satisfait un besoin grandissant d'étudiants issus de divers univers culturels; de plus en plus, ils aspirent à comprendre les rouages de l'ensemble social mystérieux avec lequel ils doivent composer et dans lequel opère le monde des affaires. Cet enseignement a également été des plus utiles dans divers cours de sciences humaines et sociales (par exemple, en travail social, en sociologie et en études régionales) où il a servi à initier les étudiants aux réalités et aux 
dimensions multiples du système social ainsi qu'aux exigences de l'intervention stratégique. La prise en compte de tous ces éléments nous paraît indispensable à la maîtrise de la profession de gestionnaire; mais elle devra aussi inspirer les autres acteurs de la vie civique formés par nos institutions d'enseignement supérieur.

\section{Notes et références}

1 Pour plus de renseignements sur la Loi sur le développement durable on consultera le texte du professeur Denis Bourque dans le présent collectif.

\section{Bibliographie}

Michel Aglietta, Laurent Berrebi, Désordres dans le capitalisme mondial, Éditions Odile Jacob, Paris, 2007.

Jacques Attali, « Pour l'économie, la crise est un tsunami qui approche », Le Devoir, 11 août 2008.

Centre d'expertise en gestion des ressources humaines ; Yves Boisvert ... [et al.]. Raisonnement éthique dans un contexte de marge de manœuvre accrue : clarification conceptuelle et aide à la décision : rapport de recherche, Québec, Secrétariat du Conseil du trésor : École nationale d'administration publique, Université Laval, 2003.

Michel Dion, Dominique Wolff, Le développement durable. Théorie et applications au management, Paris, Dunod, 2008.

Paule Haley et Christine Gagnon, «Le développement durable, une condition au bonheur ?», Revue québécoise de psychologie, vol. 28, $\mathrm{n}^{\circ} 1,159-182$.

Étienne Perrot, L'art de décider en situations complexes, Desclée de Brouwer, Paris, 2007.

John Saul, Vers l'équilibre, Petite Bibliothèque Payot, Paris, 2007.

John Saul, La mort de la globalisation, Petite Bibliothèque Payot, Paris, 2008.

Joseph E. Stiglitz, Un autre monde. Contre le fanatisme du marché, Fayard, Paris, 2006.

Muhammad Yunus, Vers un nouveau capitalisme, JCLattès, Paris, 2008. 


\section{Publicité}

\section{Doctorat en développement regional}

